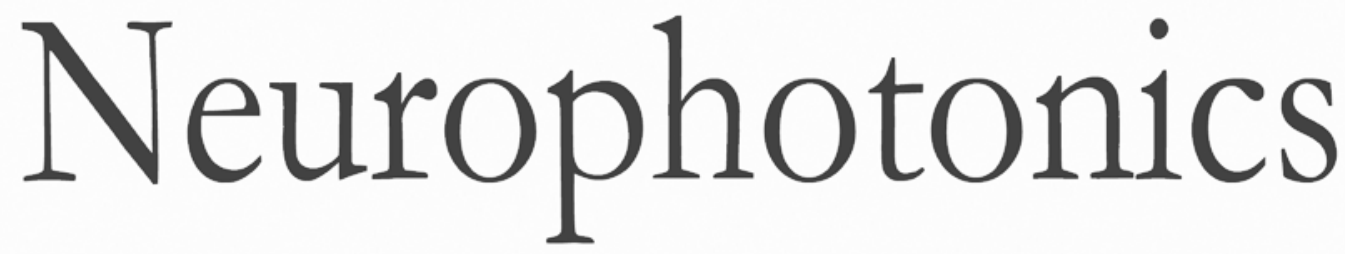

\title{
Cerebral metabolism in a mouse model of Alzheimer's disease characterized by two-photon fluorescence lifetime microscopy of intrinsic NADH
}

Carlos A. Gómez

Buyin $\mathrm{Fu}$

Sava Sakadžić

Mohammad A. Yaseen 


\title{
Cerebral metabolism in a mouse model of Alzheimer's disease characterized by two-photon fluorescence lifetime microscopy of intrinsic NADH
}

\author{
Carlos A. Gómez, ${ }^{a, \dagger}$ Buyin Fu, $^{a}$ Sava Sakadžić, ${ }^{a}$ and Mohammad A. Yaseen ${ }^{\mathrm{a}, \star}$ \\ aMassachusetts General Hospital, Harvard Medical School, Athinoula A. Martinos Center for Biomedical Imaging, Department of Radiology, \\ Charlestown, Massachusetts, United States
}

\begin{abstract}
Disruptions and alterations to cerebral energy metabolism play a vital role in the onset and progression of many neurodegenerative disorders and cerebral pathologies. In order to precisely understand the complex alterations underlying Alzheimer's disease (AD) progression, in vivo imaging at the microscopic level is required in preclinical animal models. Utilizing two-photon fluorescence lifetime imaging microscopy and the phasor analysis method, we have observed AD-related variations of endogenous fluorescence of reduced nicotinamide adenine dinucleotide (NADH) in vivo. We collected NADH FLIM images from the cerebral cortices of both APPswe:PS1dE9 mice to model amyloid $\beta$ plaque accumulation and corresponding age-matched wildtype controls. Distinct variations in NADH fluorescence lifetime between wildtype and AD mice, as well as variations related to proximity from amyloid plaques, are obvervable via the phasor method. The combination of NADH FLIM and phasor analysis allows for a minimally invasive, high-resolution technique to characterize the adverse effects of amyloid $\beta$ accumulation on mitochondrial energy metabolism and could guide our understanding of preclinical AD pathology. () The Authors. Published by SPIE under a Creative Commons Attribution 3.0 Unported License. Distribution or reproduction of this work in whole or in part requires full attribution of the original publication, including its DOI. [DOI: 10.1117/1.NPh.5.4.045008]
\end{abstract}

Keywords: Alzheimer's disease; cerebral metabolism; fluorescence lifetime imaging; microscopy; phasors.

Paper 18037R received Jul. 2, 2018; accepted for publication Nov. 30, 2018; published online Dec. $27,2018$.

\section{Introduction}

Alzheimer's disease (AD) is a devastating neurodegenerative disease with rapidly increasing incidence. Current estimates indicate that $\mathrm{AD}$ accounts for $60 \%$ to $80 \%$ of dementia cases, ${ }^{1}$ and projections estimate that it will affect as many as 74.7 million people worldwide by 2030, costing $\sim 2$ trillion for treatment and care. ${ }^{2}$ Presently, AD is diagnosed clinically when behavioral symptoms such as memory loss and deteriorating cognition manifest in patients. A "prodromal" phase of AD progression reportedly initiates as much as 20 years in advance of outwardly observable behavioral and cognitive deficiencies. Hallmark preclinical changes that begin during the prodromal phase include accumulation of soluble amyloid $\beta(\mathrm{A} \beta)$ oligomers, insoluble $\mathrm{A} \beta$ plaques, neurofibrillary tangles from misfolded tau protein, and activated microglia and astrocytes. ${ }^{3,4}$ These pathological features are associated with notable impairments to glucose metabolism, cerebral blood flow, and mitochondrial function. In addition, numerous $\mathrm{A} \beta$-induced alterations to mitochondrial structure and function have been observed. $^{5-8}$ The ability to monitor cerebral metabolism is vitally important to understanding AD's trajectory and to develop robust biomarkers and therapeutic approaches. Preclinical ADrelated changes that manifest at a cellular- and neurovascularlevel requires minimally invasive, high-resolution techniques for detailed characterization.

For several decades, measurements of reduced nicotinamide adenine dinucleotide (NADH) have been utilized as a non-

\footnotetext{
*Address all correspondence to Mohammad A. Yaseen, E-mail: mayaseen@ nmr.mgh.harvard.edu

${ }^{\dagger}$ Current Address: Boston University, Department of Biomedical Engineering,
} Boston, Massachusetts, United States destructive tool to characterize mitochondrial respiratory chain activities. NADH is an electron carrier molecule residing in almost all eukaryotic cells both in cytosol and mitochondria, and it performs vital roles in both anaerobic glycolysis and aerobic oxidative metabolism. ${ }^{9}$ Extensive studies have utilized single photon, UV excitation of NADH fluorescence in isolated mitochondria or cell suspensions as a sensitive, quantitative measure of oxygen-dependent mitochondrial respiratory chain activity. ${ }^{10-12}$ Under these in vitro conditions, oxygen levels can be adjusted and metabolic substrates can be added such that the sample's NADH can reversibly be adjusted between its fully oxidized and fully reduced states, therefore providing calibration for the minimal and maximal detectable $\mathrm{NADH}$ fluorescence signal. Confounds arising from toxicity prevent calibration and absolute quantitation in living organisms; however, evaluating relative changes of in vivo NADH fluorescence intensity still provides a useful indication of pathology-induced variations of mitochondrial function and intracellular oxygen requirements. ${ }^{13}$ The in vivo measurement techniques' utility was greatly extended by coupling NADH fluorescence with 2-photon microscopy (2PM), ${ }^{14,15}$ a powerful tool for investigating multiple facets of brain function with high spatial and temporal resolution using deeper penetrating near-infrared excitation. ${ }^{16,17}$ While fluorescence intensity imaging of NADH offers a nondestructive, label-free, and simple method to evaluate mitochondrial function, fluorescence lifetime imaging microscopy (FLIM) shares these benefits and is also insensitive to fluorophore concentration or excitation and emission intensity. By measuring the time-resolved fluorescence decay at each pixel, FLIM provides a robust method to evaluate microenvironmental conditions around a fluorophore, including changes in temperature, $\mathrm{pH}$, or in the case of $\mathrm{NADH}$, alterations in enzymatic binding. ${ }^{18,19}$ FLIM-based monitoring of NADH 
offers considerable potential for assessing more subtle variations in mitochondrial energy metabolism with subcellular resolution, particularly when it is integrated with $2 \mathrm{PM}^{20-24}$ In our previous investigations, we used an assortment of pharmacological inhibitors to validate the hypothesis that in vivo FLIM measurements can resolve disruptions to the TCA cycle, electron transport chain, and oxidative phosphorylation. ${ }^{25}$ Our earlier studies utilized traditional FLIM analysis methods based on nonlinear curve-fitting algorithms, which are computationally intensive and often exceedingly sensitive to potential inaccuracies. As an alternative to nonlinear curve-fitting methods, the polar plot (aka "phasor") analysis method offers an elegant, Fourierbased alternative approach that requires less computation power and makes no assumptions about the mathematical models of the fluorescence lifetime decays. ${ }^{26-29}$ Variations in fluorescence lifetime can easily be visualized, indicated by differences in location on the two-dimensional (2-D) phasor plot. We previously demonstrated that phasor analysis of endogenous NADH fluorescence can robustly detect pharmacologically induced alterations in mitochondrial energy metabolism. ${ }^{30}$ Shifts in the location on the phasor plots indicated disruptions to the TCA/Krebs cycle, the electron transport chain, and oxidative phosphorylation, and we found that the phasor technique enables development of improved models for classifying metabolic perturbations.

In this report, we apply two-photon fluorescence lifetime imaging microscopy (2P-FLIM) of endogenous cortical NADH for minimally invasive characterization of $\mathrm{AD}$-induced variations in cerebral metabolism in a mouse model of AD. We utilized phasor analysis to show that amyloid $\beta$ plaques cause significant variations to fluorescence lifetime of endogenous NADH. In addition, we observed AD-related variations to spatial distribution and fluorescence lifetime of the aging pigment, lipofuscin. The results show promise to guide our understanding of the metabolic alterations associated with $\mathrm{AD}$ progression.

\section{Materials and Methods}

Our imaging experiments were performed under a protocol approved by the Institutional Animal Care and Use Committee at Massachusetts General Hospital. For our studies, we utilized female transgenic APPswe:PS1dE9 mice $(N=7)$ and their age-matched wildtype controls $(N=4)$ (8 to 13 months, 22 to $30 \mathrm{~g})$. The APPswe:PS1de9 mouse model displays elevated soluble $\mathrm{A} \beta$ content and $\mathrm{A} \beta$ plaques by 4 to 6 months of age, with progressive $\mathrm{A} \beta$ accumulation thereafter. ${ }^{31}$ A 3-mm cranial window was surgically implanted into the skull and was held in place by acrylic. Mice were placed under isoflurane anesthesia and their physiological conditions (heart rate, temperature, and arterial blood gases) were monitored. $\mathrm{A} \beta$ plaques were fluorescently labeled by topical application of trypan blue. 2P-FLIM images were collected using a custom-made multimodal microscope which features a titanium:sapphire laser (Insight DeepSee, Spectra Physics, excitation wavelength tuned to $\lambda_{\mathrm{ex}}: 740 \mathrm{~nm}$, repetition frequency $f=80 \mathrm{MHz}$ ), high-numerical aperture objective lens (Olympus XLumPlan Fluor, 20×, $1.00 \mathrm{NA}, 2 \mathrm{~mm}$ working distance), galvanometer-based scanner mirrors (6215HB, Cambridge Technology, Inc.), and commercial electronics for timecorrelated single-photon counting (SPC-150, DCC,-100, GVD120, Becker \& Hickl GmbH). ${ }^{19,32}$ For these experiments, commercial SPCM software from Becker \& Hickl was used for the acquisition. Incident power varied but remained well below
$50 \mathrm{~mW}$ for all measurements. 2P-FLIM measurements consisted of repetitive $256 \times 256$ pixel raster scans performed over 80 to $150 \mu \mathrm{m}$ fields of view at frame intervals of up to $\sim 900 \mathrm{~ms}$ for $120 \mathrm{~s}$. Each recorded measurement consisted of a $256 \times 256 \times$ 256 matrix of photon counts $(x, y, t ; \Delta t \approx 50$ ps binning intervals) for NADH and trypan blue emission. A hybrid photomultiplier tube (PMT) with high detection efficiency and minimal afterpulsing (HPM-100-40 Becker \& Hickl, GmbH) was used to collect NADH emission, and trypan blue fluorescence was detected using a PMT. Compared to NADH autofluorescence (emission wavelength $\lambda_{\mathrm{em}} \sim 450 \mathrm{~nm}$ ), the emission peak for trypan blue is redshifted by over $100 \mathrm{~nm}$. We verified that trypan blue fluorescence is not seen by our NADH detection channel.

All FLIM data were analyzed using customized software developed in Matlab (Mathworks Inc., Natick, Massachusetts). By integrating the photon count matrices along the time axis, intensity images were generated and used for preprocessing. First, to isolate parenchymal NADH fluorescence, features such as blood vessels were masked out manually, as well as constituents displaying endogenous fluorescence such as lipofuscin granules. The boundaries for $\mathrm{A} \beta$ plaques were also delineated manually using the trypan blue images. Fourier-based phasor calculations were performed on the 2P-FLIM fluorescence decay $I(t),{ }^{26,27}$ in order to get the phasor coordinates $g(\omega)$ and $s(\omega)$; where angular frequency $\omega$ relates to laser repetition frequency $f$ as $2 * \pi * f$ :

$g(\omega)=\frac{\int_{0}^{\infty} I(t) \cos (\omega t) \mathrm{d} t}{\int_{0}^{\infty} I(t) \mathrm{d} t} \quad s(\omega)=\frac{\int_{0}^{\infty} I(t) \sin (\omega t) \mathrm{d} t}{\int_{0}^{\infty} I(t) \mathrm{d} t}$.

The coordinates, $g(\omega)$ and $s(\omega)$, were then plotted on a 2-D phasor map. The phasor coordinates for monoexponential decays lie along the "phasor universal circle," the semicircle defined by the equation $(g-0.5)^{2}+s^{2}=0.25 \quad(s \geq 0)$. Meanwhile fluorescence profiles that exhibit multiexponential decays will fall within the semicircle, at locations that are the vectorial sum of the fractionally weighted individual terms. These calculations were performed by our custom software along with groupwise analysis of multiple samples and comparison of different regions of interest (ROI) including concentric annular ROIs. The software also assessed separability of different phasor clusters using the Bhattacharyya distance, computed as

$D_{B}=\frac{1}{8}\left(\mu_{1}-\mu_{2}\right)^{T}\left(\frac{P_{1}+P_{2}}{2}\right)^{-1}\left(\mu_{1}-\mu_{2}\right)+\frac{1}{2} \ln \left(\frac{\left|\frac{P_{1}+P_{2}}{2}\right|}{\left|P_{1}\right|\left|P_{2}\right|}\right)$,

where $\mu_{i}$ and $P_{i}$ correspond to the mean coordinates and covariance matrix for each phasor cluster. ${ }^{33,34}$ Like the Mahalanobis distance, ${ }^{20}$ the Battacharyya distance is utilized to assess the similarity between two statistical distributions and is useful for characterizing the separability of different classes of data in multidimensional space as well as feature extraction and classification. ${ }^{35}$ It differs from the Mahalanobis distance by allowing for distributions with unequal covariance. If two distributions are identical, $D_{\mathrm{B}}$ is zero. A larger statistical distance implies greater separability between classes. We designated $D_{\mathrm{B}} \geq 0.9$ as the threshold value for significant separability between phasor clusters, as this value has been 
used to develop classification models that perform with high accuracy $(\sim 90 \%) .{ }^{36}$

\section{Results and Discussion}

We sought to evaluate AD-related alterations to mitochondrial function by measuring NADH in the living cortex of AD mouse models. Similar to flavin adenine dinucleotide (FAD), NADH is endogenous and ubiquitous within most eukaryotic cells, and it participates in multiple steps of glucose breakdown and ATP synthesis. Monitoring the fluorescence of these electron carriers uniquely enables nondisruptive assessment of energy metabolism within living biological tissue. ${ }^{9}$ However, like most intrinsic fluorophores, both FAD and NADH have low two-photon action cross sections and are thus weakly excitable compared to exogenous dyes. ${ }^{37}$ Based on our previous efforts, additional experimental optimizations are necessary to overcome limitations before FAD fluorescence can be robustly measured in living brains, including weak excitation, absorption by hemoglobin, and suboptimal detection efficiency. Consequently, our characterizations of AD-related cerebral metabolic alterations focused primarily upon lifetime-based measurements of intrinsic NADH. First, this required isolation of parenchymal NADH signal from other morphological features such as blood vessels and endogenous constituents that also displayed fluorescence. Figures 1(a) and 1(b) show example fluorescence intensity images within and around an $\mathrm{A} \beta$ plaque in the living mouse cortex from detector channels used to measure $\mathrm{NADH}$ $\left(\lambda_{\mathrm{em}}=460 \pm 30 \mathrm{~nm}\right)$ and trypan blue $\left(\lambda_{\mathrm{em}}=595 \pm 25 \mathrm{~nm}\right)$, respectively. While topical application of trypan blue enabled easy and specific visualization of $\mathrm{A} \beta$ plaques and amyloid deposits along vascular segments, the plaques were also discernible by autofluorescence in the lower wavelength emission channel, even in the absence of trypan blue. Typically, the interior portions of plaques demonstrated autofluorescence with comparable intensity to the NADH from surrounding parenchymal tissue, ${ }^{38}$ but the plaques were enveloped by a dark band around the periphery where no signal was present. The aging pigment lipofuscin was also observed in both $\mathrm{AD}$ mice and their wildtype counterparts, appearing as bright, punctate crystals with broad emission detectible in both detector channels. In the $\mathrm{AD}$ mouse model, we consistently observed the high accumulation of lipofuscin crystals around the plaque boundary, as seen in Fig. 1(a). We isolated the NADH fluorescence from neuropil and cell bodies by manually masking out ROIs corresponding to blood vessels, autofluorescence from $\mathrm{A} \beta$ plaques, and lipofuscin granules.

After masking, phasor computations were performed on all measurements and grouped using our custom software. Figures 2(a) and 2(b) show phasor clusters corresponding to parenchymal NADH and lipofuscin, respectively, in wildtype and $\mathrm{AD}$ mouse models. The clusters are displayed as contour plots of the 2-D histograms (from $40 \%$ to $100 \%$ of the histograms peak centroid). We calculated phasor coordinates corresponding to cortical tissue measurements collected in wildtype mice $(N=22$ measurements from four mice), measurements from $\mathrm{AD}$ mice around an $\mathrm{A} \beta$ plaque $(N=43$ measurements from seven mice), and measurements from $\mathrm{AD}$ mice in fields of view where no plaques were present $(N=6$ measurements from four mice). NADH measurements from all mice localized in a similar region well inside the "phasor universal circle",27,28 as our previous measurements in Sprague Dawley rats, ${ }^{30}$ supporting repeated assertions that NADH exists as a population of different enzyme-bound formulations with different fluorescence lifetimes in vivo and in vitro. Conversely, lipofuscin phasor clusters were broad and dispersed over a range of short lifetimes. For both endogenous fluorophores, phasor clusters corresponding to measurements taken from regions near $\mathrm{A} \beta$ plaques appear visually separable from measurements taken far away from plaques or within wildtype mice with no plaques. For both NADH and lipofuscin measurements, we computed the Bhattacharyya distances $\left(D_{\mathrm{B}}\right)$ between each group to assess the separability between all clusters. As seen in Fig. 2, calculated $D_{\mathrm{B}}$ distances for $\mathrm{NADH}$ around $\mathrm{A} \beta$ plaques exceeded our threshold criterion $(>0.9)$ for statistical dissimilarity from the other groups, implying significant separability of $\mathrm{NADH}$ fluorescence lifetime in the presence of $\mathrm{A} \beta$ plaques. Lipofuscin measurements from wildtype mice appear well separated from $\mathrm{AD}$ mice on the phasor plots. However, computed $D_{\mathrm{B}}$ distances fell below our designated threshold value and imply insignificant dissimilarity.

To evaluate the spatial extent of metabolic variations around $\mathrm{A} \beta$ plaques, we partitioned NADH images into concentric annular ROIs at well-defined distances around the plaque border. Figure 3(a) shows an example partitioned NADH image with (a)

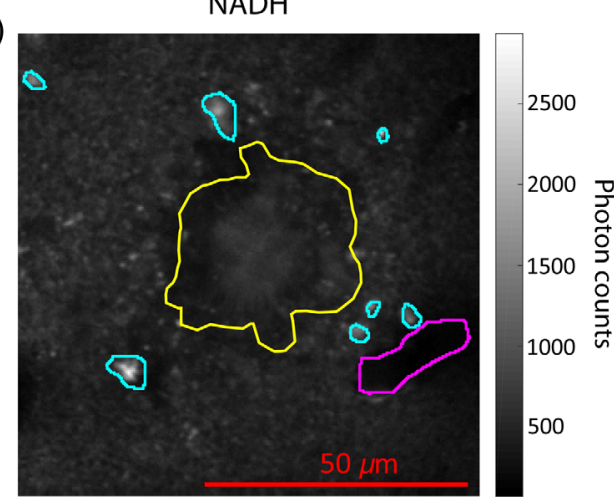

(b)

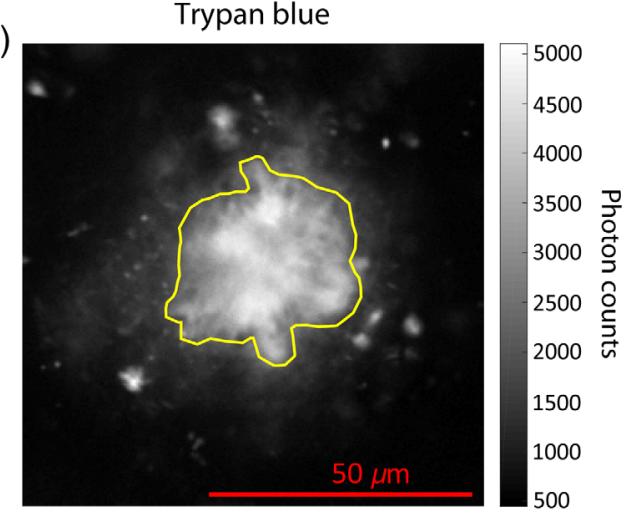

Fig. 1 Intensity image of (a) endogenous NADH fluorescence and (b) corresponding fluorecence of topically applied trypan blue, collected in vivo from cortical tissue of an AD mouse model with different morphological features outlined: yellow, amyloid $\beta$ plaque; teal, lipofuscin deposits; and magenta, cortical blood vessel. These features were masked for to isolate parenchymal NADH. 

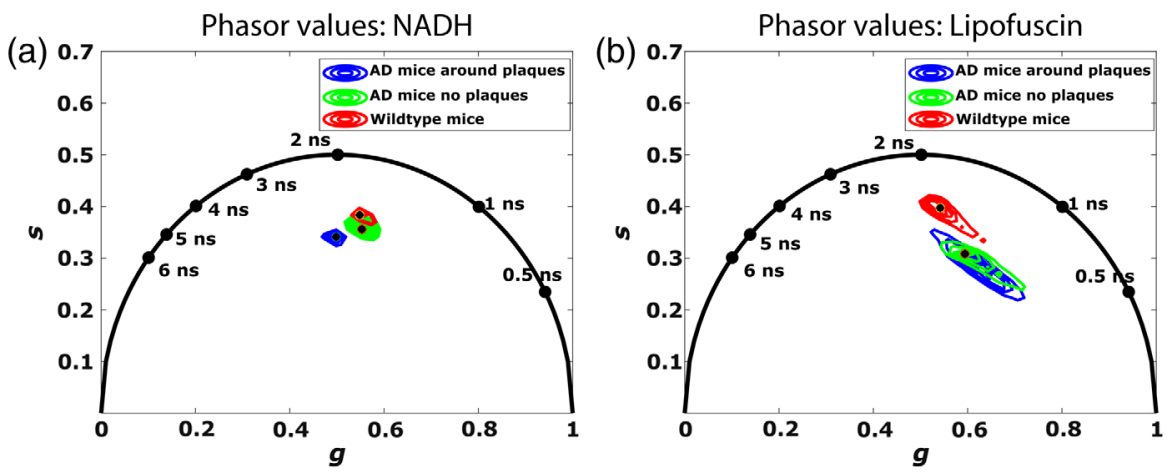

\begin{tabular}{|c|c|c|}
\hline \multicolumn{3}{|c|}{$D_{\mathrm{B}}$} \\
\hline & Wildtype & No plaques \\
\hline Around plaques & 0.947 & 0.906 \\
\hline Wildtype & -- & 0.273 \\
\hline
\end{tabular}

\begin{tabular}{|c|c|c|}
\hline \multicolumn{3}{|c|}{$D_{\mathrm{B}}$} \\
\hline & Wildtype & No plaques \\
\hline Around plaques & 0.704 & 0.073 \\
\hline Wildtype & -- & 0.375 \\
\hline
\end{tabular}

Fig. 2 Phasor clusters for the parenchymal NADH and lipofuscin collected from AD mice and wildtype controls, and computed Bhattacharyya distances $\left(D_{\mathrm{B}}\right)$ of each cluster. The NADH phasor clusters of measurements taken near $\mathrm{A} \beta$ plaques appear well separated, suggesting that phasors can be reliably used to distinguish $\mathrm{A} \beta$-related differences in cerebral metabolism. Lipofuscin measurements of wildtype mice appear distant from $\mathrm{AD}$ mouse measurements, although $D_{\mathrm{B}}$ imply similarity.
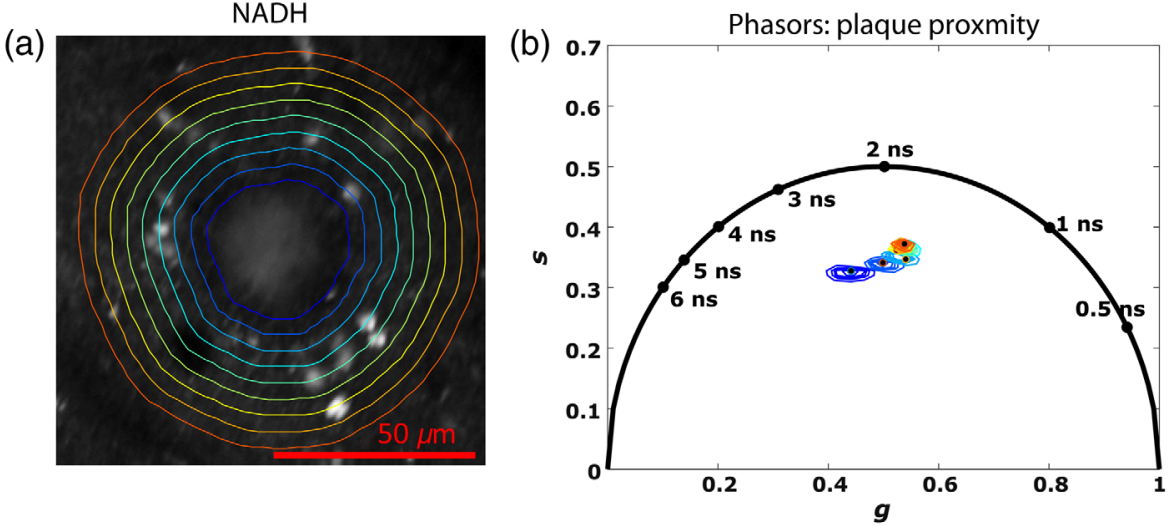

Fig. 3 (a) Intensity image of an amyloid plaque and the surrounding cortical tissue with red delineated ROIs. All ROls are 4- $\mu \mathrm{m}$ equidistant from each other. (b) Corresponding phasor clusters of the plaque (violet) and the eight cortical tissue ROls. A distinct gradient is seen in the clusters' centroids, varying with distance from the plaque.

concentric band ROIs around a plaque. Each ROI corresponds to a $4-\mu \mathrm{m}$-thick region of tissue around the plaque boundary. Figure 3(b) shows the phasor clusters computed from inside an $\mathrm{A} \beta$ plaque and the surrounding annular regions from 43 plaque measurements. As seen by the upward shift in phasor centroids of more distal ROIs, a distinct gradient persists in the phasor clusters at distances up to $36 \mu \mathrm{m}$ away from the plaque boundaries. Computed $D_{\mathrm{B}}$ values for each ROI did not meet our criterion for significant cluster separability, which suggests that the influence of $\mathrm{A} \beta$ on mitochondrial function varies continuously rather than discretely as a function of proximity to plaques. Compared to more proximal tissue measurements, NADH measurements corresponding to tissue regions near the $\mathrm{A} \beta$ plaque show lower intensity with corresponding phasor clusters that are shifted downward and closer to the origin, similar to the effects induced by application of the respiration uncoupler carbonyl cyanide-4-(trifluoromethoxy) phenylhydrazone (FCCP). ${ }^{25,30}$ At increasing distances from plaques, the phasor clusters lie in areas corresponding to relatively shorter average lifetimes, similar to those of measurements taken in wildtype mice.

Although further validation is required, the present, phasorbased observations of endogenous NADH fluorescence from living cortices of AD mouse models strongly indicate a pronounced influence of $\mathrm{A} \beta$ plaques on mitochondrial energy metabolism. Specifically, the downward left shift in phasor location and lower fluorescence intensity of NADH near plaques suggests that $\mathrm{A} \beta$ interferes with oxidative phosphorylation. These observations agree well with in vitro observations of impaired respiration and mitochondrial toxicity induced by intracellular $\mathrm{A} \beta .^{5}$ The results are also consistent with previous reports, which utilized several exogenous contrast agents to demonstrate $\mathrm{A} \beta$-induced impairments to mitochondrial prevalence and function. The dark bands surrounding plaques 
observed in our endogenous fluorescence images are in agreement with Xie et al.'s observations of mitochondrial loss near plaque borders, and our observed variations in NADH phasor clusters with plaque proximity support their observations of $\mathrm{A} \beta$ variations in mitochondrial membrane potential. ${ }^{6}$

Conversely, our observations of plaque-induced variations in lipofuscin fluorescence lifetime were unanticipated and raise intriguing questions regarding the nature and mechanisms of plaque-related toxicity. Often regarded as an aging-related pigment, lipofuscin granules are chemically and morphologically hetereogeneous masses primarily comprised of oxidatively modified protein and lipid residues that resist degradation by lysosomes. Lipofuscin is considered "biological garbage" that progressively accumulates within several tissues with age, but the rate of accumulation can be accelerated in the presence of higher oxygen or reduced by administration of antioxidants. ${ }^{39,40}$ Its presence is often overlooked or masked in brain imaging studies. Our observations of selective lipofuscin granule accumulation around plaque periphery and modest variation in fluorescence lifetime near plaques could reflect the vicious cycle of oxidative stress, mitochondrial impairments, $\mathrm{A} \beta$ accumulation, and plaque formation. ${ }^{41,42}$ Monitoring accumulation and fluorescence lifetime of lipofuscin could therefore be useful for assessing plaque formation and oxidative stress or toxicity associated with progression accumulation in the preclinical stages of AD.

Our study shows that phasor-based in vivo measurements of endogenous fluorescence are useful for characterizing distinct variations in mitochondrial metabolism and lipofuscin accumulation in the presence of $\mathrm{A} \beta$ plaques. The findings agree well with previous reports of metabolic alterations and present opportunities to noninvasively assess $\mathrm{A} \beta$-related variations in oxidative stress and plaque formation. Metabolic function is distinctly affected in regions surrounding plaques, but it is unclear whether variations in mitochondrial function and oxidative stress are entirely attributable to amyloid $\beta$. Plaques are comprised primarily of improperly cleaved amyloid $\beta$ protein, but they also contain a variety of other materials, including proteoglycans, metal ions, and inflammatory molecules such as cytokines. ${ }^{43}$ While soluble $\mathrm{A} \beta$ oligomers demonstrate notable neurotoxicity, our observations motivate further investigations to explore whether and how the additional plaque constituents contribute to metabolic impairments, oxidative stress, or autofluorescence by plaques.

\section{Disclosures}

The authors declare no potential conflicts of interest with respect to the research, authorship, and/or publication of this article.

\section{Acknowledgments}

This work was supported by NIH R00AG042026 (MAY), R01AA027097 (MAY), R01-NS057476 (SS), R01NS091230 (SS), and P01-NS055104 (SS).

\section{References}

1. Alzheimer's Association, "Alzheimer's disease facts and figures," Alzheimer's Dementia 14(3), 367-429 (2018).

2. M. Prince et al., "World Alzheimer report 2016: improving healthcare for people living with dementia: coverage, quality and costs now and in the future," 2016, http://www.alz.co.uk/ (29 June 2018).

3. B. De Strooper and E. Karran, "The cellular phase of Alzheimer's disease," Cell 164(4), 603-615 (2016).
4. M. A. Daulatzai, "Cerebral hypoperfusion and glucose hypometabolism: key pathophysiological modulators promote neurodegeneration, cognitive impairment, and Alzheimer's disease," J. Neurosci. Res. 95(4), 943-972 (2017).

5. P. M. Schaefer et al., "Metabolic characterization of intact cells reveals intracellular amyloid beta but not its precursor protein to reduce mitochondrial respiration," PLoS One 11(12), e0168157 (2016).

6. H. Xie et al., "Mitochondrial alterations near amyloid plaques in an Alzheimer's disease mouse model," J. Neurosci. 33(43), 17042-17051 (2013).

7. J. P. Cleary et al., "Natural oligomers of the amyloid-protein specifically disrupt cognitive function," Nat. Neurosci., 8(1), 79-84 (2005).

8. R. Abeti, A. Y. Abramov, and M. R. Duchen, "beta-amyloid activates PARP causing astrocytic metabolic failure and neuronal death," Brain 134, 1658-1672 (2011).

9. A. A. Heikal, "Intracellular coenzymes as natural biomarkers for metabolic activities and mitochondrial anomalies," Biomarkers Med. 4, 241-263 (2010).

10. B. Chance, B. Schoener, and V. Legallais, "Metabolically linked changes in fluorescence emission spectra of cortex," Nature 195(4846), 1073-1075 (1962).

11. B. Chance, D. Jamieson, and H. Coles, "Energy-linked pyridine nucleotide reduction: inhibitory effects of hyperbaric oxygen in vitro and in vivo," Nature 206(4981), 257-263 (1965).

12. B. Chance, "Pyridine-nucleotide as an indicator of oxygen requirements for energy-linked functions of mitochondria," Circ. Res. 38(5), 31-38 (1976).

13. A. Mayevsky and E. Barbiro-Michaely, "Use of NADH fluorescence to determine mitochondrial function in vivo," Int. J. Biochem. Cell Biol. 41, 1977-1988 (2009)

14. K. A. Kasischke et al., "Neural activity triggers neuronal oxidative metabolism followed by astrocytic glycolysis," Science 305, 99-103 (2004).

15. T. Takano et al., "Astrocyte-mediated control of cerebral blood flow," Nat. Neurosci. 9, 260-267 (2006).

16. K. Svoboda and R. Yasuda, "Principles of two-photon excitation microscopy and its applications to neuroscience," Neuron 50, 823-839 (2006).

17. J. N. D. Kerr and W. Denk, "Imaging in vivo: watching the brain in action," Nat. Rev. Neurosci. 9, 195-205 (2008).

18. W. Becker, Advanced Time-Correlated Single Photon Counting Techniques, Springer, Berlin, Heidelberg, New York (2005).

19. M. A. Yaseen et al., "In vivo imaging of cerebral energy metabolism with two-photon fluorescence lifetime microscopy of NADH," Biomed. Opt. Express 4(2), 307-321 (2013).

20. S. Osseiran et al., "Non-Euclidean phasor analysis for quantification of oxidative stress in ex vivo human skin exposed to sun filters using fluorescence lifetime imaging microscopy," J. Biomed. Opt. 22(12), 125004 (2017).

21. H. D. Vishwasrao et al., "Conformational dependence of intracellular $\mathrm{NADH}$ on metabolic state revealed by associated fluorescence anisotropy," J. Biol. Chem. 280, 25119-25126 (2005).

22. Q. Yu and A. A. Heikal, "Two-photon autofluorescence dynamics imaging reveals sensitivity of intracellular NADH concentration and conformation to cell physiology at the single-cell level," J. Photochem. Photobiol. B Biol. 95, 46-57 (2009).

23. M. C. Skala et al., "In vivo multiphoton microscopy of NADH and FAD redox states, fluorescence lifetimes, and cellular morphology in precancerous epithelia," Proc. Natl. Acad. Sci. U. S. A. 104, 19494-19499 (2007).

24. M. Evers et al., "Enhanced quantification of metabolic activity for individual adipocytes by label-free FLIM," Sci. Rep. 8, 8757 (2018).

25. M. A. Yaseen et al., "Fluorescence lifetime microscopy of NADH distinguishes alterations in cerebral metabolism in vivo," Biomed. Opt. Express 8(5), 2368-2385 (2017).

26. G. I. Redford and R. M. Clegg, "Polar plot representation for frequencydomain analysis of fluorescence lifetimes," J. Fluoresc. 15(5), 805-815 (2005).

27. M. A. Digman et al., "The phasor approach to fluorescence lifetime imaging analysis," Biophys. J. 94(2), L14-L16 (2008).

28. C. Stringari et al., "Phasor approach to fluorescence lifetime microscopy distinguishes different metabolic states of germ cells in a live tissue," Proc. Natl. Acad. Sci. U. S. A. 108, 13582-13587 (2011). 
29. J. P. Eichorst, K. Wen Teng, and R. M. Clegg, "Polar plot representation of time-resolved fluorescence," in Fluorescence Spectroscopy and Microscopy, Y. Engelborghs and A. J. W. G. Visser, Eds., Vol. 1076, pp. 97-112, Humana Press, Totowa, New Jersey (2014).

30. C. A. Gomez et al., "Phasor analysis of NADH FLIM identifies pharmacological disruptions to mitochondrial metabolic processes in the rodent cerebral cortex," PLoS One 13(3), e0194578 (2018).

31. M. Garcia-Alloza et al., "Characterization of amyloid deposition in the APPswe/PS1dE9 mouse model of Alzheimer disease," Neurobiol. Dis. 24(3), 516-524 (2006).

32. M. A. Yaseen et al., "Multimodal optical imaging system for in vivo investigation of cerebral oxygen delivery and energy metabolism," Biomed. Opt. Express 6(12), 4994-5007 (2015).

33. M. Niknazar et al., "Application of bhattacharyya distance as a dissimilarity index for automated prediction of epileptic seizures in rats," in Int. Conf. Intelligent and Advanced Systems, pp. 1-5 (2010).

34. A. Paoli, F. Melgani, and E. Pasolli, "Clustering of hyperspectral images based on multiobjective particle swarm optimization," IEEE Trans. Geosci. Remote Sens. 47(12), 4175-4188 (2009).

35. T. Kailath, "The divergence and Bhattacharyya distance measures in signal selection," IEEE Trans. Commun. Technol. 15(1), 52-60 (1967).

36. N. Nilsson, B. Hakansson, and M. Ortiz-Catalan, "Classification complexity in myoelectric pattern recognition," J. NeuroEng. Rehabil. 14, 68 (2017)
37. W. R. Zipfel et al., "Live tissue intrinsic emission microscopy using multiphoton-excited native fluorescence and second harmonic generation," Proc. Natl. Acad. Sci. U. S. A. 100, 7075-7080 (2003).

38. A. C. Kwan et al., "Optical visualization of Alzheimer's pathology via multiphoton-excited intrinsic fluorescence and second harmonic generation," Opt. Express 17(5), 3679-3689 (2009).

39. U. T. Brunk and A. Terman, "Lipofuscin: mechanisms of age-related accumulation and influence on cell function," Free Radical. Biol. Med. 33(5), 611-619 (2002).

40. A. Terman and U. T. Brunk, "Lipofuscin," Int. J. Biochem. Cell Biol. 36(8), 1400-1404 (2004).

41. A. Terman and U. T. Brunk, "Oxidative stress, accumulation of biological 'garbage', and aging," Antioxid. Redox Signal. 8(1-2), 197-204 (2006).

42. L. Zheng, J. Marcusson, and A. Terman, "Oxidative stress and Alzheimer disease: the autophagy connection?" Autophagy 2(2), 143-145 (2006).

43. C. S. Atwood et al., "Senile plaque composition and posttranslational modification of amyloid-beta peptide and associated proteins," Peptides 23(7), 1343-1350 (2002).

Biographies of the authors are not available. 